\title{
Die Reaktionen des Aluminiumnitrids.
}

\section{Von}

Fr. Fichter und Adolf Spengei.

In einer früheren Abhandlung ${ }^{1}$ wurde die Darstellung von Aluminiumnitrid aus Aluminiumpulver im Stickstoffstrom beschrieben und das Präparat durch seine Haupteigenschaften kurz charakterisiert. Bei der zunehmenden technischen Bedeutung der Verbindung schien es uns nicht uninteressant, die damaligen Versuche auszudehnen und die Reaktionen des Aluminiumnitrids möglichst vollständig zu untersuchen. ${ }^{2}$ Wir müssen dabei die allgemeine Bemerkung vorausschicken, daB in den meisten Fällen das Aluminiumnitrid nur träge und unvollkommen reagiert, indem die entstehender Produkte eine schützende Hülle um das noch unangegriffene Material bilden.

\section{Darstellung von Aluminiumnitrid aus Aluminiumpulver und Stickstoff.}

Man bekommt leider im Handel kein chemisch reines Aluminiumpulver. Ein als "chemisch-rein" bezeichnetes Präparat enthielt beispielsweise $93.07 \% \mathrm{Al}, 1.24 \% \mathrm{Fe}$ und $0.85 \% \mathrm{Si}$, ferner durch oberflächliche Oxydation gebildetes Aluminiumoxyd und einen kleinen Rückhalt des nur durch oft wiederholte Extraktion mit Äther im Soxhletapparat zu entfernenden, äußerst hartnäckig anhaftenden, von den Fabrikanten beim Pulvern zugesetzten Fettes (Talg).

Zur Darstellung von Nitrid beschickt man ein im Heraeds schen $40 \mathrm{~cm}$-Röhrenofen liegendes, $20 \mathrm{~mm}$ weites Nickelrobr mit etwa $10 \mathrm{~g}$ Aluminiumpulver, verdrängt die Luft durch sauerstofffreien, getrockneten Stickstoff, erhitzt langsam während 25 Minuten bis auf

'Z. anorg. Chem. 54 (1907), 322.

${ }^{2}$ Experimentelle Einzelheiten und Belege sind in der Basler Dissertation des Herrn Dr. A. Spenaer, „Über Alumininmnitrid und Berylliumnitrid“, München 1912, niedergelegt. 
$800^{\circ}$ und erzielt dann von etwa $820^{\circ}$ an eine lebhafte Reaktion, erkennbar am Aufleuchten der reagierenden Partien und an einer bedeutenden Erhöhung der Temperatur, die im Inneren der Reaktionsmasse in wenigen Augenblicken über $1300^{\circ}$ ansteigt, auch wenn der Heizstrom ausgeschaltet wird. Während dieser Periode starker Reaktion muB man einen kräftigen Stickstoffstrom ins Rohr leiten, wofern man nicht das Ansaugen von Luft rom andern, durch eine leere und eine mit konzentrierter Schwefelsäure beschickte Waschflasche verschlossenen Ende riskieren will. Nach kurzer Zeit ist die Hauptreaktion beendet, und das Pyrometer sinkt, während der eingeleitete Stickstoff immer reichlicher unabsorbiert entweicht. Nach dem Erkalten im Stickstoffstrom findet man zusammengesinterte harte, fast weiße Stücke von hochprozentigem Nitrid, neben grauen pulverigen unvollkommen umgewandelten Partien. Gute Präparate kann man nur durch zweimalige Behandlung mit Stickstoff erhalten, denn die gesinterten Stücke lassen das Gas nicht ins Innere dringen. Man pulvert deshalb die weißen Stücke der ersten Operation möglichst fein und behandelt nochmals während $1-2$ Stunden bei $1100-1200^{\circ}$ im Stickstoffstrom.

Wir sind auf die Darstellung des Nitrids nach diesem Verfahren (,Röhrennitrid“) deshalb näher eingetreten, weil seit unserer letzten Veroffentlichung von verschiedenen Seiten Methoden zur Gewinnung von Aluminiumnitrid beschrieben wurden, die von der unserigen etwas abweichen. Demgemäb wurden auch die Eigenschaften der Präparate verschieden angegeben.

A. J. Sofianopodtos ${ }^{1}$ ersetzt den Stickstoff durch einen trockenen Ammoniakstrom und erhält so das Nitrid als graue schwammige Masse, aus welcher er das überschüssige Aluminium durch Quecksilber entfernt. Offenbar hat er bei ziemlich niedriger Temperatur gearbeitet, denn sein Nitrid ist viel reaktionsfähiger als unser gesintertes Röhrennitrid.

KoHN-ABREsT ${ }^{2}$ hat durch Beschränkung der Temperatur auf $900^{\circ}$ im Stickstoffstrom ein dunkelgraues Präparat dargestellt, das einen niedrigeren Stickstoffgehalt aufweist als ihn die Formel AlN verlangt, und das er als ein "Subnitrid" betrachten will. Zweifellos handelt es sich um einfache Gemische von Aluminiumnitrid und freiem Aluminium. Die anfechtbaren Behauptungen von KoHN-

${ }^{1}$ Bull. Soc. chim. de France [4] 5 (1909), 614.

2 Compt. rend. Acad. Sci. Paris 1500 (1910), 918.

Z. anorg. Chem. Bd. 82. 
Arrest sind auch in einer Polemik von $\mathrm{O} . \mathrm{SERPEK}^{1}$ ins rechte Licht gesetzt worden.

DAFFY Wolk ${ }^{2}$ arbeitete nach der Methode von SofianopodLOS und gewann ein graves schwammiges Nitrid rom richtigen, der Formel AlN entsprechenden Stickstoffgehalt. Das Präparat soll sich über $1100^{\circ}$ unter Zusammensintern zersetzen. Graue Präparate verdanken ihre Färbung einem Gehalt an freiem, feinverteiltem Aluminium; man beobachtet dann oft, daB bei höheren Temperaturen das pulverige Aluminium zu kleinen Kügelchen zusammenschmilzt, was den Eindruck hervorruft, als habe eine Zersetzung unter Ausscheidung von Metall stattgefunden. Tatsächlich ist Aluminiumnitrid bis zu sebr hohen Temperaturen hinauf beständig. So verwendet beispielsweise 0 . SERPEK in seinem für die technische Herstellung von Aluminiumnitrid bestimmten elektrischen Röhrenofen ${ }^{3}$ fertiges Aluminiumnitrid als feuerfestes Futter bei Temperaturen von $1800-1850^{\circ}$, und S. A. Tuaker und H. L. ReAD ${ }^{4}$ geben an, daB erst oberhalb $2000^{\circ}$ das Nitrid Zersetzung erleide.

D. WoLk hat bei seinen Veröffentlichungen sehr viel Gewicht darauf gelegt, daß die Temperatur der Stickstoffaufnahme von uns fruher zu niedrig angegeben worden sei. Es kommt bei derartigen Messungen sehr auf die Umstände an; bei unserer Versuchsanordnung mit ziemlich großen Chargen von Aluminiumpulver steigert sich die Temperatur, wenn die Reaktion einmal begonnen hat, durch die entwickelte Wärme im Innern der Masse von selbst. Man kann übrigens schon zwischen 700 und $800^{\circ}$ eine erhebliche Bindung von Stickstoff erzielen, doch ist es schwer, die so erhaltenen Produkte auf den Maximalgehalt zu bringen. Unserer Erfahrung nach sind hochprozentige Präparate am sichersten nach obiger Vorschrift zu erzielen.

Auf einem ganz anderen Wege hat A. C. Vournasos ${ }^{5}$ Nitride gewonnen, indem er die betreffenden Metalle auf Cyanverbindungen bei hoher Temperatur einwirken läBt. Sein Aluminiumnitrid stellt ein amorphes, gelbliches Pulver dar, leicht zersetzbar durch Alkalien und Mineralsäuren.

1 Compt. rend. Acad. Sci. Paris 150 (1910), 1520.

2 Compt. rend. Acad. Sci. Paris 151 (1910), 318; Bull. Soc. chim. de Franee [4] 7 (1910), 768; Diss. Nancy 1910.

${ }^{3}$ Soc. Générale des Nitrures, D.R.P. 238340 (1910).

4 Trans. Amer. Electrochem. Soc. 22 (1912), 57.

5 Bull. Soc. chim. de France [4] 9 (1911), 506; Z. anorg. Ohem. 77 (1912), 191. 


\section{Reinigung des Aluminiumnitrids.}

Das Röhrennitrid ist stets durch freies Aluminium und Eisen grau gefärbt. Behandelt man nun dieses Präparat mit sorgfältig getrocknetem Chlorwasserstoffgas bei Rotglut, so wird zuerst das freie Aluminium und das Eisen angegriffen, indem sich die entstehenden Chloride verflüchtigen, und das Nitrid bleibt vollkommen weiß zurück. Erhitzt man längere Zeit und unter Steigerung der Temperatur bis zum Erweichen des Glases, so wird auch das Aluminiumnitrid angegriffen und unter Bildung von Aluminium- und Ammoniumchlorid verflüchtigt. Bei kurzer Behandlung aber und bei nicht allzuhoher Temperatur läßt sich die Zersetzung des Aluminiumnitrids in engen Grenzen halten. Einen Überblick über die Anreicherung im Chlorwasserstoffgasstrom geben folgende Zahlen:

$\begin{array}{ccc}\text { Rohnitrid, } & \begin{array}{c}\text { Gewichtoverlust } \\ \text { Stickstoffgehalt in } \%\end{array} & \begin{array}{c}\text { Stickstoffgehalt } \\ \text { im HCl-Strom in } \%\end{array} \\ 31.71 & 4.83 & \text { nach der Behandlung in \% } \\ 31.71 & 4.29 & 32.43 \\ 32.79 & 2.62 & 33.65 \\ 32.79 & 4.56 & 33.65\end{array}$

\section{Analyse des Aluminiumnitrids.}

Aluminiumnitrid wird durch Wasser zersetzt nach der Gleichung

$$
\mathrm{AlN}+3 \mathrm{H}_{2} \mathrm{O}=\mathrm{Al}(\mathrm{OH})_{3}+\mathrm{NH}_{3} \text {, }
$$

doch verläuft der ProzeB selbst bei lebhaftem Kochen und reichlichen Wassermengen nur langsam, weil die entstehende Tonerde das unzersetzte Nitrid einhullt. So wurden beispielsweise bei zweistündigem Kochen und Abdestillieren (4.5 ccm per Minute) nur 43.4\% des vorhandenen Stickstoffs abgespalten.

Auch die viel rascher verlaufende Zersetzung durch wässerige Alkalien bedarf einer ziemlich langen Zeit zur quantitativen Durchführung, so daB sie als analytische Methode nicht zu empfehlen ist.

Sehr prompt gelingt die Zersetzung durch Schmelzen mit Kaliumhydroxyd. Wir konstruierten zu diesem Zweck einen kleinen Apparat aus einem kurzen, unten geschlossenen Stahlrohr von $35 \mathrm{~mm}$ innerem Durchmesser, auf das ein aufgeschliffener Bronzedeckel mit Hilfe einer Überwurfmutter angepreßt wird. Im Deckel befindet sich ein weites Gasableitungsrohr, und ein engeres, fast bis auf den Boden des Stahlzylinders reichendes Röhrchen, das zum Durchsaugen von Luft am SchluB der Analyse dient. Man verschlieBt das obere 
Ende des Röhrchens mit einem Gummischlauch und Quetschhahn, gibt $2.5 \mathrm{~g}$ gröblich zerkleinertes Stangenkali in den Stahlzylinder, darauf rasch die abgewogene Nitridmenge und nochmals $2.5 \mathrm{~g}$ Kali, verschraubt sofort, verbindet das Gasableitungsrohr durch einen Reitmayeraufsatz mit zwei Péligotröhren, die abgemessene $1 / 2$-norm. Salzsäure enthalten, und erhitzt. Schon nach 5 Minuten ist das Ammoniak auf Grund der Reaktion

$$
\mathrm{AlN}+3 \mathrm{KOH}=\mathrm{K}_{3} \mathrm{AlO}_{3}+\mathrm{NH}_{3}
$$

ausgetrieben, und nach dem Öffnen des Quetschbahnes wird nun noch während 5 Minuten Luft durchgesaugt. Die ganze Analyse ist in 15 Minuten beendet, und die Methode empfiehlt sich durch diese Raschheit sehr. Doch hat man mit mangelhafter Dichtigkeit des Verschlusses zu kämpfen, und das Stahlrohr wird durch die Kalischmelze leck. Wir haben deshalb diese Methode mit einer zuverlässigeren wenn auch langsamer arbeitenden vertauscht.

Alle wăsserigen Säuren wirken auf Aluminiumnitrid ein nach der allgemeinen Gleichung

$$
\mathrm{AlN}+4 \mathrm{HX}=\mathrm{AlX}_{8}+\mathrm{NH}_{4} \mathrm{X} .
$$

Für die Analyse empfiehlt sich am meisten die Anwendung einer mäBig verdünnten Schwefelsäure; konzentrierte Schwefelsäure wirkt sehr unvollständig wegen der Unlöslichkeit des Aluminiumsulfats. Mit einer Mischung von $10 \mathrm{ccm}$ konzentrierter Schwefelsäure und $40 \mathrm{ccm}$ Wasser wird das Nitrid im Kjeldahlkolben 5-6 Stunden gelinde gekocht, wodurch Lösung oder Umwandlung in z. T. ungelöstes Sulfat eintritt. Man versetzt darauf mit überschüssiger Lauge und destilliert wie bei einer Kjeldahlanalyse das Ammoniak ab (wir verwandten hierzu mit Vorteil den von KRIEGER ${ }^{1}$ angegebenen Apparat). Sowohl die AufschlieBung als die Destillation können fast ohne Aufsicht durchgeführt werden, so daB der gröBere Zeitaufwand gegenüber der Kalischmelze nicht allzusehr ins Gewicht fällt.

Nach der zweiten Methode analysierten wir sämtliche von uns dargestellten Röhrennitride mit folgendem Ergebnis:

$\begin{array}{lllllllll}\text { Präparat Nr. } & 1 & 2 & 3 & 4 & 5 & 6 & 7\end{array}$

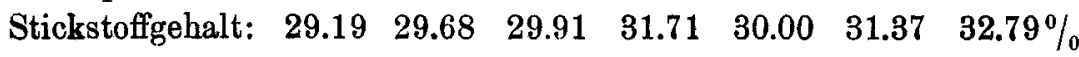

Reines AlN müBte $65.92 \%$ Al und $34.08 \% \mathrm{~N}$ enthalten; das beste Präparat Nr. 7 weist gegenüber dem theoretischen Wert einen

1 Chem.-Ztg. 35 (1911), 1063. 
Mindergehalt von $1.29 \%$ Stickstoff auf. Die oben erwähnte Reinigung mit Chlorwasserstoff erhöhte den Gehalt auf $33.65 \%$, was gegenüber dem theoretischen Wert nur noch einen Fehlbetrag von $0.43 \%$ bedeutet. Bedingt ist diese Differenz durch das im Aluminiumpulver enthaltene Aluminiumoxyd und Silicium.

Im AnschluB an dieses Kapitel sei auch das Verhalten des Aluminiumnitrids gegen andere wässerige Säuren geschildert.

Am raschesten wirkt konzentrierte wässerige Flußsäure, die in $4 \frac{1}{2}$ Stunden auf dem Wasserbad völlige Zersetzung des Nitrids herbeiführte.

Wässerige Salzsäure (gleiche Volumina konzentrierter HCl und Wasser) löst beim Kochen im Kjeldahlkolben das Nitrid in 12 bis 15 Stunden bis auf ein paar Flöckchen (Tonerde) auf.

Konzentrierte Salpeters äure erzielte in 11 Stunden noch keine vollständige AufschlieBung; doppeltnormale Salpetersäure dagegen ergab nach 8 stündigem Kochen im Präparat Nr. 229.59 statt $29.68 \% \mathrm{~N}$.

5-norm. Brom wassersto ffsäure gab nach 21 stündigem Kochen 29.22 statt $29.68 \% \mathrm{~N}$ in demselben Präparat. Verdünntere Säure war bedeutend weniger wirksam.

Verdünnte Hexachloroplatinisäure $(2 \%)$ gab beim Kochen mit Aluminiumnitrid Ammoniumchloroplatinat.

Man weiß aus der Patentliteratur, ${ }^{1}$ daß auch die Lösungen von leicht hydrolysierbaren Salzen auf Aluminiumnitrid zersetzend einwirken. In der Tat konnten wir bei 16 stündigem Kochen mit $2 \%$ iger Ferrichloridlösung unter Ausscheidung von gelbem Eisenoxydgel $2 / 3$ des vorhandenen Stickstoffs als Ammoniumchlorid abspalten. Eine 3\% ige Alaunlösung erwies sich als weniger wirksam.

\section{Aluminiumnitrid and Wasserstoff.}

Aluminiumnitrid und Wasserstoff reagieren nicht miteinander. Ebensowenig ist Aluminiumnitrid imstande, die Vereinigung von Stickstoff und Wasserstoff katalytisch zu beeinflussen, oder Ammoniak zu zerlegen.

5. Aluminiumnitrid und Halogene.

Gesintertes, bei hohen Temperaturen dargestelltes Röhrennitrid wird von den Halogenen nur langsam bei höherer Temperatur an-

${ }^{1}$ Bad. Anilin- u. Sodafabrik, D.R.P. 235868 (1909). 
gegriffen. Man muß allerdings dabei auf sorgfältigste Trocknung der Gase achten, denn bei Gegenwart von Feuchtigkeit tritt eine sehr lebhafte, von Feuererscheinung begleitete Reaktion ein.

In trockenem luftfreiem Chlor vollzieht sich bei etwa $760^{\circ}$ der Zerfall in Aluminiumchlorid und elementaren Stickstoff nach

$$
2 \mathrm{AlN}+3 \mathrm{Cl}_{2}=2 \mathrm{AlCl}_{3}+\mathrm{N}_{2} \text {. }
$$

Diese Reaktion verläuft langsamer und schwerer als der Angriff des freien Metalls in grauen Präparaten, so daB bei der Chlorierung wie bei der Behandlung mit trockenem Chlorwasserstoffgas eine Anreicherung des Rückstandes zu erzielen ist. Bei 1 stündiger Behandlung in heller Rotglut fand beispielsweise ein Gewichtsverlust von $29.51 \%$ statt, während der weißgewordene Rückstand einen Stickstoffgehalt von 32.35 gegenüber $31.71 \%$ im Ausgangsmaterial aufwies.

Trockenes Brom wirkt noch weniger auf Aluminiumnitrid ein als Chlor. Nach 1 stündiger Behandlung in heller Rotglut betrug der Gewichtsrerlust nur $1.90 \%$, während gleichzeitig eine Anreicherung von 31.71 auf $32.29 \% \mathrm{~N}$ im weibgewordenen Rückstand eingetreten war.

Ein stärkerer Angriff als mit Chlorgas ist dagegen zu erzielen durch einen Strom von Schwefelchlorürdampf, ${ }^{1}$ der aus einer im Ölbad erwärmten Waschflasche enwickelt und mit Hilfe von Kohlendioxyd über das im Verbrennungsrohr erhitzte Nitrid geleitet wird. Das Aluminiumnitrid verflüchtigt sich dabei bis auf einen unbedeutenden Rest vollständig unter Bildung von Stickstoff und Aluminiumchlorid.

\section{Aluminiumnitrid und Oxydationsmittel.}

In der ersten Abhandlung wurden die Schwierigkeiten geschildert, die beim Versuch der Verbrennung des Aluminiumnitrids in der kalorimetrischen Bombe auftraten; sie bestanden darin, daB die Reaktion mit Sauerstoff nie quantitativ durch die ganze Masse hindurch erfolgen wollte, obschon die Verbrennung im Sauerstoffstrom nach

$$
4 \mathrm{AlN}+3 \mathrm{O}_{2}=2 \mathrm{Al}_{2} \mathrm{O}_{3}+2 \mathrm{~N}_{2}
$$

bei genügend hoher Temperatur vollständig verläuft. Allerdings vollzieht sich auch im strömenden Sauerstoff die Oxydation nur langsam,

1 Vgl. C. Matianon und F. Bounion, Compt. rend. Acad. Sci. Paris 138 (1904), 631 . 
indem die entstehende Tonerdehülle das eingeschlossene Nitrid vor weiterer Einwirkung schützt.

Luft wirkt von $760^{\circ}$ an merklich ein; bei $840^{\circ}$ war das Präparat völlig weiß geworden, enthielt aber immer noch $16 \%$ Stickstoff, entsprechend $47 \%$ Nitrid. Reiner Sauerstoff reagiert nicht viel lebhafter als Luft: nach 4 stündigem Erhitzen auf $830-840^{\circ}$ enthielt der Rückstand noch $8 \%$ Stickstoff, entsprechend $23.5 \%$ Nitrid.

Wir suchten nun nach Oxydationsmitteln, die imstande wären, in prompter Reaktion die Verbrennung des Aluminiumnitrids zu veranlassen. Am wirksamsten ist Kaliumbichromat; ein inniges Gemisch molekularer Mengen von AlN und $\mathrm{K}_{2} \mathrm{Cr}_{2} \mathrm{O}_{7}$ gibt beim Erhitzen rote Dämpfe, und der Rückstand enthält keinen Nitridstickstoff mehr, gibt aber Nitratreaktion.

Bleichromat ist fähig in analoger Anordnung wie sie zur Bestimmung des Stickstoffs nach Dumas dient, den Stickstoff des Aluminiumnitrids quantitativ abzuspalten. Wir fanden so in einem Präparat 28.92 statt $29.6 \% \mathrm{~N}$.

Eine Mischung von zwei Teilen Natriumperoxyd und ein Teil Aluminiumnitrid reagiert nach kurzem Erhitzen unter Feuererscheinung, doch pflanzt sich die Reaktion nicht durch die ganze Masse hindurch fort; man bekommt stets nitridhaltige Rückstände, während andererseits der abgespaltene Stickstoff zu Nitrat oxydiert wird. Bariumperoxyd reagiert nicht.

Bleioxyd reagiert unter Feuererscheinung und Bildung von Bleikügelchen; der Rückstand enthält noch Nitrid. Bleidioxyd ist weniger wirksam als Bleioxyd. Oxydationsmittel von basischem Charakter sind überhaupt eher befähigt, Aluminiumnitrid anzugreifen, als solche von indifferenter oder saurer Natur. Dementsprechend bleiben Kaliumnitrat, Kaliumchlorat, Kaliumpermanganat, Kupferoxyd und Cerdioxyd ziemlich wirkungslos.

\section{Alumininmnitrid, schwefel and Schwefelverbindungen.}

Schwefeldampf wirkt im offenen Verbrennungsrohr auf erhitztes Aluminiumnitrid nicht ein; wenn man aber ein Gemenge von Schwefel und Aluminiumnitrid in ein widerstandsfähiges Glasrohr einschlieBt und auf $380-400^{\circ}$ erhitzt, so nimmt der Stickstoffgehalt der Präparate ab (von 31.71 bis auf $29.59 \%$ ), während gleichzeitig nach sorgfältiger Entfernung des freien Schwefels bis zu $6.8 \%$ 
gebundener Schwefel nachzuweisen ist. Eine Reaktion etwa im Sinne der Gleichung

$$
4 \mathrm{AlN}+3 \mathrm{~S}_{2}=2 \mathrm{Al}_{2} \mathrm{~S}_{3}+2 \mathrm{~N}_{2}
$$

ist um so wahrscheinlicher, als $\mathrm{W}$. BILIz ${ }^{1}$ nachgewiesen hat, daB Aluminiumsulfid, in einer Stickstoffatmosphäre bis zu $2100^{\circ}$ erhitzt, wohl Schwefel abspaltet unter Bildung von Monosulfid, aber keinen Stickstoff aufnimmt. Bei Fortsetzung unserer Versuche unter Anwendung höherer Temperaturen $\left(550-600^{\circ}\right)^{2}$ hatten wir viel Schwierigkeiten durch das Explodieren der Röhren, so daB wir die Reaktion einstweilen nicht vollständiger durchführen konnten.

Schwefelkohlenst offdampf wirkt bei Rotglut, wo er selbst z. T. zersetzt ist, ähnlich wie Schwefel unter partieller Verdrängung des Stickstoffs. Doch geht die Reaktion nur sehr langsam und hatte nach 10 tägiger Behandlung noch nicht ein Viertel des Nitrids umgewandelt; gleichzeitig schied sich reichlich Kohle ab, und gelegentlich bildeten sich kleine, irisierende, in Tetrachlorkohlenstoff lösliche Kriställchen (Schwefelstickstoff?).

Schwefelwasserstoffgas ist auch bei höheren Temperaturen fast wirkungslos; ebenso Schwefeldioxyd.

\section{Aluminiumnitrid, Phosphor und Phosphorverbindungen.}

Sowohl beim Erhitzen von Aluminiumnitrid auf Rotglut in einem mit Phosphordampf beladenen Wasserstoffstrom, als auch bei 8 stïndigem Erhitzen von Nitrid und Phosphor im Druckrohr auf $520^{\circ}$ kann man den Stickstoff zu etwa ein Fünftel durch Phosphor verdrängen. Dagegen ist Phosphortrichloriddampf bei heller Rotglut obne Einwirkung.

\section{Alnminiumnitrid, Kohle, und Kohlenstoffverbindungen.}

Aluminiumnitridpulver, innig gemischt mit $R \mathrm{u} B$, erleidet beim Erhitzen bis auf $1220^{\circ}$ im Wasserstoffstrom keine Veränderung. ${ }^{3}$ Ebensowenig tritt eine Reaktion zwischen Aluminiumkarbid und Stickstoffgas bei Temperaturen bis $1200^{\circ}$ ein. Wir haben die Absicht, diese Versuche bei höheren Temperaturen fortzusetzen, um die Frage endgültig aufzuklären, ob bei der technischen Darstellung

1 Z. anorg. Chem. 71 (1911), 182.

2 A. Stock und F. Gomolka, Ber. 42 (1909), 4515.

${ }^{9}$ Auch Bor und Silicinm sind bei $1000^{\circ}$ wirkungslos. 
des Aluminiumnitrids das Aluminiumkarbid als Zwischenprodukt eine Rolle spielt oder nicht.

Kohlenoxyd ist bei $1100^{\circ}$ ohne Einwirkung auf Aluminiumnitrid.

Kohlendioxyd wirkt auf Aluminiumnitrid bei hoher Temperatur einfach oxydierend. Bei $1040^{\circ}$ trat noch keine Gewichtsvermehrung ein, von $1120^{\circ}$ an wurde die Oxydation deutlich nachweisbar, und bei $11 / 2$ stündigem Erhitzen auf $1140^{\circ}$ wurden $54.14 \%$ des Nitrids in Oxyd rerwandelt. Bei $1300^{\circ}$ gelang die Umwandlung bis $\mathrm{zu} 89 \%$; der Stickstoffgehalt sank von 29.68 auf $3.92 \%$ herunter. Eine gleichzeitige Bildung von Cyanverbindungen, wie sie BRIEGLEB und GEUTHER ${ }^{1}$ bei analoger Reaktion mit Magnesium. nitrid beobachtet haben, war nicht eingetreten.

Dagegen gelingt die Überführung des Nitridstickstoffs in Cyanidstickstoff unter etwas anderen Bedingungen.

Mischt man überschüssiges Aluminiumnitrid mit trockenem Alkalikarbonat, so erhält man beim Erhitzen im Porzellantiegel kleine Mengen von Cyanid; dies erinnert an die Beobachtung von F. WöHLER ${ }^{2}$ am Bornitrid, das mit Kaliumkarbonat Metaborat und Cyanat, oder bei Überschuß von Bornitrid Cyanid liefert.

Eine reichlichere Bildung von Cyanid erfolgt aber nur, wenn man Aluminiumnitrid mit einer völlig wasserfreien Mischung gleicher Teile Natriumkarbonat und Kaliumkarbonat unter Zusatz von RuB oder Kohlepulver in einer Kohlenoxydatmosphäre auf $750-800^{\circ}$ erhitzt. Aus einer großen Reihe von Versuchen greifen wir folgendes Beispiel heraus:

$0.2505 \mathrm{~g}$ AlN (Präp. Nr. 7 mit $32.79 \%$ N) wurde mit $3 \mathrm{~g}$ SodaPottaschemischung und $0.3 \mathrm{~g} \mathrm{RuB} \mathrm{im} \mathrm{Heraeus} \mathrm{schen} \mathrm{Ofen} 13 / 4$ Stunden lang im Kohlenoxydstrom auf $800^{\circ}$ erhitzt; die erhaltene Schmelze wurde ausgelaugt und im Filtrat das Cyanion als Silbercyanid gefällt; so ergab sich eine Umwandlung ron $30.4 \%$ des Nitridstickstoffs. Durch übermäBige Erhöhung der Menge der Karbonatmischung kann die Ausbeute noch gesteigert werden.

$\ddot{A}$ thylalkohol wirkt auf Aluminiumnitrid erst bei $230^{\circ} \mathrm{im}$ zugeschmolzenen Glasrohr ein; diese Temperatur liegt sehr nahe beim kritischen Punkt, und man hat darum mit häufigen Explosionen zu kämpfen. Die Umsetzung betrifft nach 8-9stündigem Erhitzen von

1 Ann. d. Chem. 123 (1862), 235.

${ }^{2}$ Gmelis-Kradt-Frigdhem I. III, (1911), S. 439, nach der Gleichung: $\mathrm{BN}+\mathrm{K}_{2} \mathrm{CO}_{3}=\mathrm{KBO}_{\mathbf{2}}+\mathrm{KCNO}$. 
$0.5 \mathrm{~g}$ Nitrid (mehr darf man nicht nehmen!) mit $10 \mathrm{ccm}$ Alkohol etwa $6 \%$ des Nitridstickstoffs, der sich in Form einer tertiären, gegen salpetrige Säure beständigen organischen Base in der Lösung findet. Der Vorgang entspricht offenbar der Gleichung

$$
\mathrm{AlN}+3 \mathrm{C}_{2} \mathrm{H}_{5} \cdot \mathrm{OH}=\mathrm{Al}(\mathrm{OH})_{3}+\left(\mathrm{C}_{3} \mathrm{H}_{5}\right)_{3} \mathrm{~N} \text {. }
$$

Magnesiumnitrid wird von Alkohol nicht angegriffen, ${ }^{1}$ Calciumnitrid gibt Ammoniak und Calciumäthylat. ${ }^{2}$

\section{Vergleich des Röhrennitrids mit dem technischen Nitrid.}

Das gesinterte Röhrennitrid läßt unter dem Mikroskop keine Kristallisation erkennen. Wir verdanken aber Herrn Dr. O. SfRPeE einige Präparate von technisch dargestelltem Aluminiumnitrid, das prächtig glänzende, weiße hexagonale ${ }^{3}$ Kristalle bildet.

Die beiden Stoffe stimmen nun in ihren chemischen Reaktionen vollkommen überein. So lieb sich beispielsweise das technische Nitrid beim Kochen mit mässig verdünnten Säuren gerade so aufschließen wie das gesinterte Röhrennitrid. Wir fanden in zwei Versuchen nach dem oben geschilderten Verfahren mit Schwefelsäure einen Stickstoffgehalt von 31.22 und $31.30 \%$ und stellten einen Parallelversuch mit Röhrennitrid und technischem Nitrid durch Kochen mit verdünnter Salzsäure an, wobei nach 9 Stunden beide gleich schweren Proben genau gleich weit (zu fast $99 \%$ ) zersetzt waren. Ebenso zeigte es sich, daß technisches Nitrid beim Erhitzen im Schwefelchlorürdampf rasch in Stickstoff und Aluminiumchlorid zerfällt. Da bei dieser Behandlung auch Aluminiumoxyd angegriffen wird, so erfuhr das technische, mit Oxyd verunreinigte Nitrid eine Anreicherung, indem der Stickstoffgehalt des Rückstandes von 31.3 auf $32.9 \%$ anstieg.

Der Hauptunterschied zwischen den beiden Präparaten - ab. gesehen von der Kristallgestalt - liegt im spezifischen Gewicht. Die Bestimmungen, die teils mit Wasser, teils mit Toluol durchgeführt wurden, ergaben als Mittelwerte bei Zimmertemperatur
Röhrennitrid, $32.8 \%$ N, 3 Messungen, 2.88, kristallisiertes Nitrid, $31.3 \% \mathrm{~N}, 4$ Messungen, 3.18.

Die Bestimmungen sind nur vorläufige, weil keines der beiden Präparate als chemisch reines Aluminiumnitrid gelten darf. Der Unter-

1 O. EMmerung, Ber. 29 (1896), 1635.

2 H. Morssav, Compt. rend. Acad. Sci. Paris 127 (1898), 497.

3 E. Bronnert, Bull. Soc. Industr. Mulhouse 79 (1909), 39. 
schied im spezifischen Gewicht liegt innerhalb der Grenzen, die man häufig bei mehr oder weniger hoch erhitzten, schwer schmelzbaren anorganischen Stoffen findet.

\section{Zusammenfassung.}

Die Darstellung von Aluminiumnitrid aus Aluminiumpulver im Stickstoffstrom wird genau beschrieben.

Das rohe, durch freies Aluminium verunreinigte "Röhrennitrid“ kann durch kurzes Erhitzen im gut getrockneten Chlorwasserstoffgasstrom weiB und reiner erhalten werden.

Zur Analyse kann entweder die Kalischmelze in einem geeigneten Apparat oder die KJELDAHL sche Methode mit mäBig verdünnter Schwefelsäure dienen. Letztere ist zuverlässiger.

Das Verhalten des Aluminiumnitrids beim Behandeln mit wässerigen Säuren, mit Wasserstoff, mit Halogenen, mit Oxydationsmitteln, mit Schwefel und Schwefelverbindungen, mit Phosphor und Phosphorverbindungen, mit Kohlenstoff und Kohlenstoffrerbindungen wird untersucht.

Die chemische Identität des amorphen gesinterten Röhrennitrids mit einem technisch dargestellten, schön kristallisierten Aluminiumnitrid wird auf Grund verschiedener Reaktionen bewiesen, dagegen ein Unterschied der beiden Präparate im spezifischen Gewicht festgestellt.

Basel, Anorganische Abteil. der Chem. Anstalt, April 1913.

Bei der Redaktion eingegangen am 23. April 1913. 